Line Fischer

Université de Namur - Institut IRDENA (Belgique)

France Dantinne

Université de Namur - Institut IRDENA (Belgique)

Évelyne Charlier

Université de Namur - Institut IRDENA (Belgique)

\section{Évaluation des effets d'un dispositif de formation initiale visant à susciter la réflexivité chez les futurs enseignants}

\author{
Evaluation of the effects of an initial training scheme \\ designed to induce reflexivity among preservice teachers
}

$\mathbf{R}$

ésumé

Cet article présente une analyse des composantes de la réflexivité qui émergent de l'évaluation faite par des futurs enseignants d'un dispositif mis en place en formation initiale, au sein de l'Université de Namur, en Belgique. Ce dispositif, fondé sur I'analyse des pratiques, vise à susciter la réflexivité.

La perception de ses effets a été récoltée par l'entremise des questionnaires. L'analyse qualitative des données a permis d'étudier les composantes de la réflexivité évoquées lors de

l'évaluation du dispositif par les apprenants. Cette recherche évaluative vise à aider les formateurs à réguler le dispositif suivant les objectifs de

formation.

Mots-clés

Réflexivité, analyse des pratiques, formation initiale des enseignants, processus réflexif.

Abstract

This paper presents an analysis of the reflexivity components that emerge from the evaluation of an initial teacher training scheme at University of Namur, in Belgium, by preservice teachers. The training scheme, based on practice analysis, seeks to induce learner reflexivity. Written surveys were used to gather the student teachers' perceptions of the scheme's effects. Qualitative data analysis

was applied to investigate which reflexivity components are found in their evaluation of the training scheme. The purpose of this evaluative research is to help trainers adjust the scheme based on training objectives.

Keywords

Reflexivity, practice analysis, initial teacher training, reflexivity process.

\section{Introduction}

\section{Contexte de l'étude}

De nos jours, les métiers se complexifient, se nuancent et se spécifient. Les contextes sociaux, économiques, politiques et technologiques participent à cette complexification. Ces environnements de travail en redéfinition permanente supposent que les professionnels développent des compétences nouvelles, et notamment la capacité d'apprendre à partir de l'analyse de leurs pratiques ainsi que la capacité à adopter une posture réflexive.

La réflexivité est plus que jamais une démarche de développement professionnel et identitaire individuelle et collective, qui peut favoriser l'adaptation de l'individu à son environnement de travail. De nombreuses formations visent à la favoriser (Donnay et Charlier, 2006) dans différents champs professionnels : éducation (Charlier et al., 2013; Tardif, Borges et Malo, 2012; Vacher, 2015), santé (Ghaye, 2006), psychologie (Scaife, 2010)... Les dispositifs de formation sont nombreux et variés et utilisent volontiers la dimension collective pour promouvoir la réflexivité grâce à des méthodologies diverses.

Le présent article se fonde sur l'analyse de données issues d'un dispositif d'analyse des pratiques mis en place au sein de la formation initiale des enseignants de l'enseignement secondaire, à l'Université de Namur, en Belgique. Ce dispositif vise à faire émerger une posture réflexive chez les futurs enseignants. Dans la suite de cet article, ce qui sera mis en lien avec la "posture réflexive " de l'étudiant futur enseignant se réfèrera à l'apparition des différentes composantes réflexives décrites ci-après. 
Cette recherche vise en particulier à examiner en quoi le dispositif d'analyse des pratiques mis en place favorise la réflexivité des étudiants, et ce, par l'investigation des composantes de la réflexivité évoquées par les futurs enseignants lors de leur évaluation des effets du dispositif.

Voici donc les questions qui ont guidé notre recherche : quels sont les effets du dispositif identifiés par les apprenants? Quelles composantes de la réflexivité sont exprimées lors de leur évaluation du dispositif? Ces questions ont pour but l'émergence d'une réflexion au sein de l'équipe des formateurs afin de permettre la régulation du dispositif.

\section{La formation initiale des enseignants en Belgique francophone}

En Belgique francophone, afin d'obtenir le titre d'agrégé de l'enseignement secondaire supérieur (niveau $7 \mathrm{du} \mathrm{CEC}^{1}$ ), il est nécessaire de suivre 300 heures de formation initiale à l'université. Cette formation de 30 crédits se réalise au terme d'un master (agrégation) ou au cours d'un master (finalité didactique).

En Belgique, la formation initiale des enseignants vise le développement de 13 compétences selon le décret D. 08-02-2001². Ce décret mentionne que la formation initiale des enseignants doit permettre la professionnalisation des enseignants (Wittorski, 2008) par le développement de l'identité professionnelle (Maroy, 2001) et la réflexivité (Schön, 1983). Des dispositifs visant à initier à la réflexivité sont donc en cohérence avec les objectifs fixés par les textes légaux.

Toutefois, la création de dispositifs visant à susciter la réflexivité des futurs enseignants en formation initiale à l'Université de Namur a également pris racine dans trois constats relatés par les formateurs de ces étudiants. En effet, ils observaient des difficultés régulières chez les futurs enseignants : a) à faire face à des situations professionnelles complexes; b) à articuler les savoirs théoriques et la réalité de terrain (notamment dans les stages); c) à s'insérer professionnellement. Dans le but de rendre les futurs enseignants « mieux adaptés » à leur future profession et de les aider à dépasser ces difficultés, différents dispositifs de formation visant à susciter la réflexivité ont été mis sur pied par chaque enseignant dans ses propres activités d'enseignement. De plus, un dispositif commun à différents cours a été créé dans une collaboration interdisciplinaire, c'est l'évaluation de celui-ci par les étudiants qui fait l'objet de cet article.

Après avoir décrit brièvement le contexte de cette recherche et l'intérêt d'analyser des dispositifs visant à susciter la réflexivité en formation initiale, le cadre théorique sera abordé et suivi de la description du dispositif. Le point suivant s'attachera à expliciter la méthodologie adoptée dans cette recherche (recueil et traitement des données). Les résultats seront ensuite passés en revue. Pour finir, la discussion sera présentée ainsi que la conclusion, les limites et les perspectives pour de futures recherches.

\section{Cadre théorique et conceptuel}

Nous présentons ici en premier lieu nos référents théoriques sur la réflexivité. Tout d'abord, nous aborderons le lien entre réflexivité et analyse des pratiques. Ensuite, nous développerons en quoi la réflexivité se distingue, selon notre position, de la réflexion. Ensuite, les composantes de la réflexivité retrouvées dans la littérature seront passées en revue. Le dispositif en question fera l'objet du point 3. 
Pour commencer, la relation qui unit l'analyse des pratiques et la réflexivité est présentée. Les pratiques sont définies ici comme « des manières habituelles d'agir, qui de par leur récurrence, constituent des réponses aux variations des éléments du contexte situationnel moins labiles que celles relevant de l'action ou de l'activité » (Trinquier, 2014, p. 222). L'analyse des pratiques constitue depuis plusieurs années une des sources privilégiées de savoirs dans le champ de l'éducation, comme le rappellent Donnay et Charlier (2006). Elle propose une démarche systématique et explicite de création de savoirs, intégrant des savoirs issus des pratiques et des savoirs issus du monde scientifique. Elle constitue donc une tentative de réduction du fossé perçu par les enseignants entre les savoirs pratiques utilisables en classe et les savoirs académiques, considérés comme peu transférables en situation (Donnay et Charlier, 2006).

Quels liens peut-on maintenant faire entre analyse des pratiques et réflexivité? Tout d'abord, il est nécessaire de rappeler que l'analyse des pratiques dépasse le cadre de la réflexivité, car certains dispositifs d'analyse des pratiques ne visent pas à faire développer une posture réflexive (même si c'est le cas au sein du nôtre). Ensuite, dans le cas de notre dispositif, la posture réflexive aiderait le praticien à faire émerger ses pratiques professionnelles, à les rendre conscientes et à les expliciter. Elle permettrait également au sujet de générer une nouvelle forme de savoir sur ses pratiques, un savoir sortant de l'expérience singulière et devenant transférable et communicable à autrui (c'est le «saut épistémologique » dont parlent Donnay et Charlier, 2006). Ce nouveau savoir permettrait au final une meilleure compréhension de ses pratiques, l'évolution de celles-ci ainsi que davantage de « prise » sur les situations professionnelles. Buysse et Vanhulle (2009) ajoutent que le praticien réflexif utiliserait la réflexion sur et dans l'action en vue de résoudre les problèmes se posant dans l'exercice de son métier ou en vue d'être conscient des solutions qu'il apporte. Par conséquent, la réflexivité est ici assimilée davantage à une posture personnelle permettant la création de savoirs chez le praticien, par opposition à l'analyse des pratiques, davantage considérée comme une démarche ou une méthodologie témoignant de l'intention du formateur. Cette posture de réflexivité permettrait au professionnel de tirer parti de ses propres expériences et ainsi de pouvoir s'inscrire une démarche de développement professionnel à long terme.

Ensuite, il est nécessaire de distinguer la réflexivité de la réflexion. Comme le rappelle Perrenoud (2004), même l'action la plus routinière implique des opérations mentales de haut niveau. Il ne suffit donc pas de témoigner d'une pensée qui oriente et régule l'action pour être réflexif. Dans notre acception, « réfléchir », c'est « mettre en mental », adopter une position méta par rapport à une situation spécifique, grâce à un système de représentations (souvent grâce au langage). Mais qu'en est-il alors de la réflexivité? Selon notre position, c'est, en premier lieu, l'objet de la réflexion qui permet de séparer réflexion et réflexivité. En effet, un praticien réflexif se prend comme objet de sa réflexion, il se regarde agir comme dans un miroir et se questionne sur le «comment» et " pourquoi » il agit (Perrenoud, 2004). En second lieu, la pratique réflexive se différencie de la simple réflexion par le fait qu'elle nécessite la mobilisation des savoirs, des théories et des concepts. En effet, la réflexivité implique le passage de données spécifiques, intuitives... à une tentative de formalisation des matériaux et des observations, c'est-à-dire à une analyse basée des concepts (Perrenoud, 2004). C'est le « saut épistémologique » dont parlent Donnay et Charlier (2006). Quant à la réflexion, « elle serait propre à la personne qui réfléchit et non transférable à d'autres situations ou d'autres personnes» (Donnay et Charlier, 2006, p. 62), le saut épistémologique n’y serait donc pas présent. 
Plus qu'un simple découpage théorique, cette distinction amène des implications pratiques, notamment en termes d'enjeux de formation des enseignants. En tant que formateur, susciter la réflexivité s'avère une tâche bien plus complexe que susciter la réflexion. La compétence réflexive nécessite en effet pour l'acteur d'adopter une mise à distance de la situation, s'en extrayant mentalement ainsi qu'une prise de recul sur lui-même. Pour Schön (1994), l'enseignant réflexif passe d'une réflexion dans l'action à une réflexion dans l'après-coup, lui permettant de relire la situation et de la transformer en connaissances. Ce saut épistémologique permettrait à l'acteur de se décentrer de son propre point de vue et de la situation précise.

Après avoir différencié réflexivité et réflexion, nous présenterons maintenant certaines composantes qui nous semblent essentielles à la réflexivité. Au sens de Schön (1994), la réflexivité permettrait au praticien d'adopter une posture d'extériorité. Cette posture permettrait d'une part, de se distancer de la situation, de la regarder de l'extérieur pour essayer de l'objectiver et d'autre part, de réaliser un retour sur soi-même, se regarder agir en situation. Pour Perrenoud (2004), la posture réflexive est engagée quand le praticien « analyse son analyse » comme s'il s'agissait de l'action ou de la pensée d'autrui. Cela nécessite donc de se regarder penser et agir, c'est-à-dire de se dissocier pour un moment de sa propre pensée. Cette posture d'extériorité peut être facilitée par un tiers, qu'il s'agisse d'une ou de plusieurs personnes, de théories, de concepts ou de grilles de lecture (dans notre dispositif, le rôle de l'Autre ainsi que le recours à des théories « communicables » semblent faciliter cette posture). Par conséquent, tout praticien réfléchit donc à sa pratique, mais cela ne signifie pas pour autant qu'il engage la réflexivité, définie comme une «sorte de réflexion sur la réflexion sur les pratiques. Il y aurait, dans la réflexivité, un saut épistémologique propice à une théorisation des pratiques et une prise de conscience de soi dans la situation »(Donnay et Charlier, 2006, p. 64). Ainsi, la mise à distance de la situation permise par la réflexivité place le sujet dans une position d'analyste-observateur de la situation et de lui-même (quiest-ce qui se joue dans cette situation dont je suis un élément?). L'enseignant réaliserait donc un retour sur lui-même en se prenant comme objet de réflexivité. Cela engage qu'il puisse opérer un retour sur ses propres pratiques en situation (comment expliquer quion en soit arrivé là avec cette classe? Comment aurais-je pu être perçu en agissant autrement?) et qu'il puisse construire du savoir généralisable sur luimême grâce à la posture qu'il adopte (je me rends compte que lorsque j'agis comme ça...; je réalise que quand cette pensée me traverse...).

\section{Description du dispositif d'analyse des pratiques à destination des futurs enseignants}

Le dispositif dont les effets sont évalués par les futurs enseignants est ici décrit. Il s'agit donc d'un dispositif d'analyse des pratiques portant sur l'analyse de vidéos d'anciens étudiants stagiaires en situation de classe. Il s'inscrit au sein d'un ensemble de dispositifs visant à pallier les difficultés présentées ci-dessus et à susciter la réflexivité chez les futurs enseignants. Lintention de développer des compétences réflexives chez les étudiants est donc présente en amont, mais aussi en aval de ce dispositif, et ce, au moyen des différentes activités de formation s'étalant tout au long de l'année.

Ce dispositif précis touche une centaine d'étudiants par année et prend la forme d'une séance se déroulant au milieu de l'année académique (cette séance est précédée d'une séance préparatoire durant la première partie de l'année visant à familiariser les étudiants à une partie de la démarche). La séance principale regroupe les futurs enseignants par quinze, mais ils travaillent également en sous-groupes 
selon les phases de l'activité. La particularité de cette séance est qu'elle est animée conjointement par un didacticien et un psychopédagogue, et ce, durant les quatre heures de séance. En effet, à l'origine, ce dispositif a vu le jour il y a six ans grâce à la volonté des didacticiens et psychopédagogues de cette formation initiale de créer une activité transversale, intégrant leurs intentions pédagogiques et permettant de mettre la complémentarité de leurs expertises au profit des étudiants. Il faut noter que l'interdisciplinarité est aussi présente au sein des cohortes d'étudiants puisqu'ils proviennent de didactiques disciplinaires variées (biologie, économie, mathématiques...).

Plus précisément, la séance se fonde sur l'analyse de séquences d'enseignement filmées durant les stages d'anciens étudiants en formation. En effet, l'utilisation de vidéo dans la formation d'enseignants débutants apparait comme un moyen privilégié de renforcer le lien entre la formation et la pratique de stage puisquelle permet de «capturer » la richesse et la complexité des situations de classe (Gaudin et Chaliès, 2012). Le format vidéo permettrait donc de mieux préparer les étudiants aux problématiques professionnelles du métier (Ria et Leblanc, 2011). Selon Ria (2018), la vidéoformation leur fournirait également plusieurs modalités d'appropriation et de transformation de leur activité professionnelle selon les designs utilisés, ayant des effets différents (dans notre cas, en se reconnaissant dans l'activité d'autrui).

Cette analyse se déroule selon quatre étapes, qui correspondent aux compétences visées par le dispositif et sont annoncées aux étudiants. Quant à la réflexivité, elle en est la finalité, mais n'est pas un objectif explicité comme tel aux étudiants. Aucune définition théorique de la réflexivité et de ses composantes, ni même de l'analyse des pratiques n'a été fournie aux étudiants, et ce, volontairement. En effet, l'hypothèse des formateurs est qu'elle sera amorcée par l'entremise de l'analyse des pratiques de l'enseignant sur la vidéo ainsi que par les discussions guidées par les formateurs et partagées au sein des groupes (la réflexivité ne peut se penser en dehors d'un objet auquel elle s'applique, objet qui est ici fourni par l'analyse des pratiques d'un enseignant sur vidéo).

Quatre compétences sont donc valorisées au sein du dispositif d'analyse des pratiques : la capacité à décrire, à problématiser, à analyser les pratiques et à construire des pistes d'action (également visées par les autres dispositifs de la formation). Les étapes préconisées pour l'analyse des pratiques sont les suivantes :

1) Les étudiants sont invités à visionner la séquence vidéo à domicile et à décrire ce qu'ils observent en s'en tenant le plus possible aux faits (travail individuel).

2) En séance, chaque étudiant partage sa description et crée une question de problématisation y étant liée. Ces questions sont présentées au groupe (temps individuel puis collectif). Dans le cadre de ce dispositif, problématiser signifie identifier un point d'entrée pour traiter une situation (Charlier et al., 2013) et, dans ce cas, formuler une question sur l'angle choisi.

3) Ensuite, les étudiants sont placés en sous-groupes et choisissent une question de problématisation par groupe afin de procéder à l'analyse, réalisée sous forme d'un schéma sur une affiche (temps collectif).

4) Pour finir, des pistes d'action sont construites par sous-groupes. L'analyse et les pistes sont présentées aux autres groupes par oral grâce à l'affiche et un partage est réalisé avec le restant du groupe (temps collectif). 
De manière générale, ce dispositif mise sur la dimension collective par l'intervention de l'Autre (le collègue, le groupe, le formateur) dans l'analyse de la pratique. En effet, le présupposé des formateurs est que la présence et l'interaction avec autrui dans ce cadre favorisent une décentration de son propre point de vue, nécessaire à la prise de distance centrale dans la réflexivité.

\section{Méthodologie}

\section{Recueil des données}

Dans le cadre de cette étude, nous tentons prioritairement d'analyser les effets perçus du dispositif d'analyse des pratiques par les futurs enseignants, c'est-à-dire d'examiner la manière dont les étudiants vivent un dispositif désigné par les formateurs comme " réflexif ».

Tout d'abord, notre démarche méthodologique a consisté à récolter les données d'évaluation du dispositif par les étudiants. Cette évaluation a été réalisée avec un questionnaire écrit, composé de six questions ouvertes. Ces questions sont formulées de manière large afin d'orienter le moins possible les réponses (par exemple : quel[s] apprentissage[s] retenez-vous de la séance?).

Ce questionnaire a été administré à l'ensemble des étudiants ayant participé au dispositif. Ceux-ci forment un groupe hétérogène (ils ont de 21 à 50 ans environ; ils sont issus de différentes didactiques; ils ont déjà enseigné pour certains, ils sont en réorientation pour d'autres; une majorité d'entre eux en sont à leur premier contact avec la profession enseignante). Le questionnaire a été distribué en fin de séance et a été rempli à ce moment, de manière anonyme. Au total, 68 questionnaires ont été remplis.

Il s'agira donc d'une recherche évaluative, dans le sens de Rutman (1982) qui la présente comme un processus dans lequel le chercheur applique une méthodologie scientifique afin de rassembler des données fiables et valides pour examiner en quoi des activités particulières produisent des effets ou des résultats.

\section{Traitement des données}

Les données ont ensuite été traitées de manière qualitative. Les questionnaires ont été lus par deux chercheuses de manière indépendante une première fois. Une seconde lecture a eu pour but de faire émerger les réponses les plus fréquentes au sein des données pour les différentes questions. Ensuite, les chercheuses ont créé de manière indépendante des catégories sur base des idées les plus fréquemment retrouvées au sein des réponses et ont comparé les catégories émergentes. N'ont été retenues que les catégories présentes sous une forme similaire tant chez l'une que chez l'autre chercheuse. Ces catégories ont été nommées par les chercheuses, sur base du vocabulaire utilisé par l'étudiant ou sur base d'une reformulation la plus fidèle possible à l'idée exprimée 3 .

Douze catégories ont ainsi été créées et numérotées. Les quatre premières catégories se rapportent aux quatre compétences déclarées dans le dispositif. Les catégories suivantes se rapportent à des effets supplémentaires relevés par les étudiants et sont au nombre de huit selon la catégorisation opérée par les chercheuses. Afin de faciliter la compréhension des catégories par le lecteur, la grille de codage qui a été créée est présentée ci-dessous (Tableau 1). Le numéro et le titre de chaque catégorie sont placés dans la colonne de gauche du tableau. La catégorie est exemplifiée par un verbatim ${ }^{4}$ dans la colonne de droite. Les différentes familles de catégories ${ }^{5}$ sont expliquées sous leur titre. 


\section{Tableau I}

Grille de codage des données

\begin{tabular}{|c|c|}
\hline Catégories émergentes & Exemples de verbatims issus des réponses aux questionnaires \\
\hline \multicolumn{2}{|l|}{$\begin{array}{l}\text { Compétences évoquées } \\
=\text { ces catégories se base } \\
\text { d'analyser et d'élargir les }\end{array}$} \\
\hline 1. Description & "C'est surtout compliqué de décrire sans émettre de jugement vis-à-vis de ce qui est observé» \\
\hline 2. Problématisation & $\begin{array}{l}\text { "J'ai mieux compris que la problématisation n'était pas le fait de trouver un problème mais de le } \\
\text { formuler sous un angle plus théorique» }\end{array}$ \\
\hline 3. Analyse & $\begin{array}{l}\text { "Le dispositif permet un développement de la capacité à analyser une situation en détail et d'élargir } \\
\text { ses angles de vue» }\end{array}$ \\
\hline 4. Proposition de pistes d'action & «On peut penser à davantage de pistes d'action si on décrit sans juger » \\
\hline \multicolumn{2}{|c|}{$\begin{array}{l}\text { Prise de conscience/explicitation } \\
=\text { ces catégories émergent de réponses d'étudiants exprimant une prise de conscience à propos d'un objet (la relativité des points de vue, l'importance } \\
\text { de l'interaction avec les collègues, l'ancrage de toute situation dans un contexte spécifique...). }\end{array}$} \\
\hline 5. De la relativité des points de vue & $\begin{array}{l}\text { "La perception de la situation est différente chez chacun. Selon sa personnalité, on n'observe pas les } \\
\text { mêmes choses. Et au-delà de l'observation, on ne les interprète pas de la même manière» }\end{array}$ \\
\hline $\begin{array}{l}\text { 6. De l'importance d'analyser ses } \\
\text { pratiques }\end{array}$ & $\begin{array}{l}\text { "L'analyse de soi est une partie intégrante de la méthode, on apprend à se " connaitre », à connaitre } \\
\text { sa façon de penser » }\end{array}$ \\
\hline 7. De l'importance de l'interaction & $\begin{array}{l}\text { "Léchange est enrichissant et permet la remise en question de notre manière de penser, de décrire, } \\
\text { impliquant une bonne prise de distance» }\end{array}$ \\
\hline 8. De ses préconceptions & $\begin{array}{l}\text { "Quelques préconceptions et normes qui me sont propres n'avaient jamais été explicitées, aussi la } \\
\text { force et la présence de ces préconceptions» }\end{array}$ \\
\hline 9. De la complexité du métier d'enseignant & $\begin{array}{l}\text { "Certaines situations peuvent paraitre anodines et peuvent pourtant receler pas mal de } \\
\text { problématiques différentes» }\end{array}$ \\
\hline 10. Distanciation par rapport aux normes & "Il n'existe pas une seule bonne réponse à une situation donnée» \\
\hline $\begin{array}{l}\text { 11. De de la contextualisation de la } \\
\text { situation }\end{array}$ & "lly a une notion d'équilibre, d'adaptation aux contextes et aux classes» \\
\hline \multicolumn{2}{|c|}{$\begin{array}{l}\text { Intentions de transfert } \\
=\text { cette catégorie relate des intentions d'utilisation des apprentissages de la séance vers des situations professionnelles }\end{array}$} \\
\hline 12. Intentions d'action & "Ça pourra mê̂tre utile pour mieux objectiver la situation d'un élève » \\
\hline
\end{tabular}




\section{Résultats}

Cette rubrique vise à présenter les résultats obtenus à la suite du traitement des données. Le tableau ci-dessous reprend chaque catégorie et présente ensuite le nombre total d'étudiants qui évoquent au moins une fois cette catégorie au sein de leur questionnaire (par exemple, 45 étudiants ont évoqué la compétence de description au sein de leur questionnaire). La présence de la catégorie est codée pour chaque étudiant de manière binaire au sein de l'ensemble du questionnaire (présente au sein du questionnaire $=1$, absente $=0$ ). Par conséquent, dans le cas où cette même catégorie apparait plusieurs fois chez un même étudiant, elle compte uniquement pour 1 point.

Les cinq catégories évoquées (Tableau 2) par un plus grand nombre d'étudiants sont les suivantes ${ }^{6}$ : compétence de description, prise de conscience de ses préconceptions, prise de conscience de l'importance de l'interaction, compétence d'analyse et prise de conscience de la contextualisation de la situation. C'est celles-ci qui feront l'objet de la discussion.

\section{Tableau 2}

Catégories éroquées par les étudiants en terme de fréquence

\begin{tabular}{l|c}
\hline Catégories émergentes & $\begin{array}{l}\text { Nombre d'étudiants } \\
\text { qui évoquent au } \\
\text { moins une fois la } \\
\text { catégorie dans le } \\
\text { questionnaire }\end{array}$ \\
\hline 1. $\quad$ Description & 45 (I) \\
\hline 2. $\quad$ Problématisation & 3 \\
\hline 3. $\quad$ Analyse & 37 (IV) \\
\hline 4. $\quad$ Proposition de pistes d'action & 14 \\
\hline 5. $\quad$ Prise de conscience de la relativité des points de vue & 9 \\
\hline 6. $\quad$ Prise de conscience de l'importance d'analyser ses pratiques & 18 \\
\hline 7. $\quad$ Prise de conscience de l'importance de l'interaction & 40 (III) \\
\hline 8. $\quad$ Prise de conscience de ses préconceptions & 41 (II) \\
\hline 9. $\quad$ Prise de conscience de la complexité du métier d'enseignant & 7 \\
\hline 10. $\quad$ Prise de conscience par rapport aux normes & 9 \\
\hline 11. $\quad$ Prise de conscience de la contextualisation de la situation & 36 (V) \\
\hline 12. Intentions d'action & 30 \\
\hline
\end{tabular}




\section{Discussion}

En partant de l'idée que la prise de conscience et de recul sur soi ainsi que la distanciation de la situation sont centrales à la réflexivité, les catégories pouvant référer à ces composantes seront présentées en premier lieu $(8,1,3)$. En second lieu, le rôle de l'Autre permettant au praticien de se décentrer de son propre point de vue sera abordé dans la continuité de ces composantes réflexives insistant sur l'importance de la posture d'extériorité (par rapport à soi et à la situation). La catégorie "prise de conscience de l'importance de l'interaction " y sera liée. (7). Pour finir, ce qui apparait au sein de la littérature comme "la conversation avec la situation" (Schön, 1994) sera relié à la catégorie de " prise de conscience de la contextualisation de la situation » (11) insistant sur la capacité du praticien réflexif à pouvoir analyser chaque situation de manière située.

Si l'on se réfère à la définition de la réflexivité selon Donnay et Charlier (2006), elle serait une « sorte de réflexion sur la réflexion sur les pratiques. Il y aurait, dans la réflexivité, un saut épistémologique propice à une théorisation des pratiques et une prise de conscience de soi dans la situation » (p. 64). Si l'on découpe cette définition, nous pouvons en abstraire les idées principales dont certaines sont exprimées par les étudiants au sein du matériau. En premier lieu, la réflexivité impliquerait une prise de conscience de soi dans la situation, ce qui nous semble proche de la catégorie " prise de conscience de ses préconceptions (8) » dans laquelle les étudiants mentionnent une prise de recul sur eux-mêmes ("La séance m'a permis de faire émerger mes préconceptions, de les comprendre ainsi que celles des autres: sontelles les mêmes ou différentes? "). Ensuite, selon Donnay et Charlier (2006), la réflexivité comprendrait un saut épistémologique effectué par le sujet qui passerait alors d'un langage personnel, singulier et peu communicable à autrui à un langage fondé sur des connaissances, des concepts "stabilisés » et donc appréhendables par autrui. Il faut noter qu'il ne s'agit pas forcément de savoirs scientifiques, mais des savoirs partagés, qui sortent donc de l'expérience spécifique à un individu. Cette progression de l'individu d'un type de savoirs à un autre fait penser à la catégorisation de Van der Maren (1993) sur les savoirs enseignants. En effet, l'auteur différencie cinq types de savoirs enseignants : le savoir savant, appliqué, stratégique, la praxis et le savoir artisan. Dans le cadre de cette activité, selon cette typologie, nous pourrions inférer que les étudiants opèrent une transition d'un savoir qui se rapproche du savoir " artisan » (savoir qui ne s'explique pas, qui s'apprend dans l'action) à un savoir " praxis ». Le «savoir praxis» est un discours de réflexion des enseignants sur la pratique, qui est le résultat d'une analyse prospective ou rétrospective. Il est « un savoir communicable impliquant une médiation, un recul, une altérité » (Van der Maren, 1993, p. 162). Il faut noter qu'il est seulement possible de faire l'hypothèse de la présence de ce saut épistémologique à travers les données récoltées par le choix et le type de langage utilisé par les étudiants au sein des questionnaires. Elle devrait cependant être vérifiée grâce à des données complémentaires.

Comme décrit dans le cadre théorique, selon Donnay et Charlier (2006, p. 62), « la réflexivité fait donc adopter une posture d'extériorité au praticien dont la distanciation prend deux formes, une mise à distance de la situation par la prise de position d'observateur-analyste et une prise de recul sur soi, car le praticien se prend comme objet de réflexivité ». Cette mise à distance de la situation par l'observation et l'analyse apparait dans les verbatim appartenant aux compétences de description (1) et d'analyse (3) ("il est très difficile de se focaliser sur les faits en restant objectif »; " la séance permet d'apprendre à prendre du recul pour analyser une situation plus en profondeur »). La prise de recul sur soi est observée comme 
expliqué précédemment. Les deux formes de distanciation liée à la posture d'extériorité du praticien réflexif semblent donc également présentes au sein des données récoltées.

Ensuite, le rôle de l'altérité permettant au praticien réflexif d'" analyser son analyse " (Perrenoud, 2004) comme s'il s'agissait de l'action/pensée d'autrui et donc de faciliter une posture d'extériorité nous semble apparaitre au sein des données. Le nombre d'étudiants évoquant «l'importance de l'interaction (7) » en témoigne. Le rôle de l'Autre semble être favorablement perçu par les étudiants au sein du dispositif puisqu'il permettrait «la prise de conscience de la relativité de son point de vue » et "qu'il n'y a pas une seule bonne manière d'être enseignant ». Bertone, Chaliès et Clot (2009) observent même au sein de données empiriques issues d'un programme de recherche sur les pratiques réflexives en formation, que l'activité collective et les échanges en formation constituent une ressource pour les enseignants novices leur permettant d'analyser autrement leur activité professionnelle. La confrontation à l'Autre semble donc aider dans la prise de conscience de la multiplicité des réponses possibles face à une problématique, dans la prise de recul par rapport à son propre point de vue et dans son analyse. Ceci rejoint l'idée de Perrenoud (2005) sur le praticien réflexif qui ne "réfléchit pas pour réfléchir", mais vise plus d'efficacité et de cohérence dans sa pratique professionnelle. Il a donc besoin des autres pour confronter ses observations et analyses. Comme le soulignent Yvon et Clot (2003), l'enseignant novice, en devenant l'analyste de sa propre pratique en dialogue avec autrui, réalise ce qu'il n'a pas pu ou voulu réaliser en situation. Il faut noter que l'Autre est également une condition inhibant l'apprentissage dans la perception de certains étudiants qui témoignent de la difficulté à travailler en groupe sur le contenu de la vidéo et à créer du «savoir » ensemble. En bref, l'hypothèse émise par les formateurs selon laquelle l'Altérité au sein du dispositif favoriserait la réflexivité est partiellement corroborée par les données (cette altérité n'étant enrichissante que sous certaines conditions).

Pour terminer, l'idée que tout professionnel doit posséder certaines ressources afin de pouvoir être flexible selon le contexte afin de pouvoir « converser avec la situation » (Schön, 1994) émerge également au sein des données. En effet, la catégorie « conscience de la contextualisation de la situation (11) »'y rapporte. Les idées des étudiants classées au sein de celle-ci se rapportent à l'importance de l'adaptation $\mathrm{du}$ professionnel à chaque situation ("il faut s'adapter à la classe que l'on a en face de nous"; "on gère des classes différentes de manière différente»). Selon Schön, la " conversation avec une situation» est " une activité mentale de haut niveau, qui mobilise, du moins lorsque le problème l'exige, de multiples ressources pour trouver une solution originale » (1994, cité dans Perrenoud, 2004, p. 35). Comme le souligne également Perrenoud (2004, p. 35), « un raisonnement professionnel [...] fait appel à une forme d'intuition, de création, de bricolage, à partir de la science, mais aussi de l'expérience et de l'expertise du praticien ».

En conclusion, différentes composantes de la réflexivité peuvent être mises en lien avec certaines catégories émergeant des réponses des étudiants aux questionnaires. De manière générale, ces réponses témoignent d'abord de prises de conscience par rapport à soi, aux situations et à la complexité du métier d'enseignant. Si l'on part de l'idée que la réflexivité permet l'adoption d'une posture d'extériorité, le dispositif mis en place en constitue peut-être une première étape, celle de la prise de conscience, c'est-à-dire d'une première découverte par le sujet des normes qui le guident, des valeurs qui le dirigent, des représentations qui le contrôlent et des attentes qu'il fait peser sur lui-même et sur les autres. Prendre conscience, c'est déjà créer une distance entre ses actes et ce qui les gouverne. Plus précisément, 
ces prises de conscience peuvent, selon nous, faciliter l'adoption d'une posture d'extériorité du sujet face à lui-même et aux situations auxquelles il est confronté ainsi que l'aider à rendre ses pratiques explicites et à les théoriser.

Ce recueil de données ainsi que la confrontation des verbatim des étudiants aux définitions de la réflexivité permet donc d'identifier les composantes de la réflexivité qui émergent à la suite de l'évaluation de ce dispositif par les apprenants Cela semble informer le formateur sur ce qui est retenu de manière saillante ${ }^{7}$ chez les futurs enseignants à la suite du dispositif ainsi que sur les apports du dispositif pour les apprenants (selon ce qu'ils en disent). Plus largement, il amène le formateur à se questionner sur le dispositif et sur les conditions mises en place pour susciter la réflexivité afin de pouvoir le réguler si besoin est.

\section{Conclusion et perspectives}

À travers cette recherche évaluative, nous avons cherché à observer quelles composantes de la réflexivité étaient évoquées par les étudiants dans l'évaluation du dispositif. Après une analyse qualitative des données, nous avons constaté que certains effets perçus par les étudiants pouvaient se rapporter à des composantes de la réflexivité, certaines ne pouvant néanmoins être étudiées par la méthodologie adoptée. D'abord, les futurs enseignants évoquent une prise de conscience de leurs préconceptions, rapprochée de la prise de recul sur soi dans la réflexivité. Selon les données analysées, ils tendent à se prendre comme objet de leur propre réflexion. En outre, la mise à distance de la situation apparait également au sein des effets identifiés par les étudiants (description et analyse de la pratique). Ensuite, le rôle de l'Autre est largement exprimé au sein des verbatim. L'Autre permettrait notamment la prise de conscience de la relativité de son propre point de vue, mise en lien avec la prise de recul sur soi qu'implique la réflexivité, mais cela uniquement sous certaines conditions, qu'il sera nécessaire d'étudier davantage. Pour finir, l'importance de la capacité d'adaptation de l'enseignant à la singularité de toute situation de classe, nommée « conversation avec la situation » (Schön, 1994) est également mise en lien avec les données.

L'étude présente par ailleurs certaines limites. Tout d'abord, bien que certaines de ces composantes réflexives soient mises en avant par les apprenants, leur articulation reste à préciser: comment s'articulent-elles au niveau conceptuel ainsi que dans le vécu de l'étudiant au sein de ce dispositif? De plus, le formateur pourrait aussi se demander quelles sont les conditions du dispositif favorisant ces composantes réflexives. Des entretiens avec les étudiants ayant vécu ce dispositif pourraient permettre d'éclairer davantage ces interrogations.

Dans un second temps, cette recherche visait à se focaliser sur les effets perçus par les étudiants à la suite de la participation au dispositif. Au cours de nos recherches, il nous est apparu clairement qu'il était difficile de pouvoir identifier les effets de la réflexivité en les distinguant du processus réflexif. Si nous posons l'hypothèse que le processus réflexif est itératif, cette distinction théorique et pratique semble, en effet, difficile. Notre méthodologie n'a pas permis de répondre à cette question puisqu'il nous semble que le moment où l'on a choisi pour récolter les données (en cours de formation) nous a donné accès à certaines informations qui peuvent, en réalité, plutôt constituer des processus d'apprentissage de la réflexivité que des effets. 
En outre, le dispositif est adressé à des étudiants qui possèdent déjà un vécu professionnel pour certains et ont sans doute commencé à développer des attitudes réflexives, par opposition à d'autres étudiants pour lesquels il s'agit du premier contact avec le métier. Il y a fort à parier que ce dispositif d'analyse des pratiques visant à faire développer une posture réflexive prenne donc des sens différents chez ces deux catégories d'étudiants. L'effet de celui-ci n'est sans doute pas analogue non plus, initiateur de réflexivité pour certains, renforçateur du processus réflexif pour d'autres. Nous émettons donc l'hypothèse que les données récoltées peuvent révéler plutôt des processus ou des effets selon le vécu de l'étudiant et particulièrement son expérience professionnelle préalable.

Une autre limite de cette étude concerne les effets du dispositif et se rapporte à l'idée précédente. En effet, il est impossible d'attester que les effets récoltés au sein de cette recherche soient uniquement des effets liés au dispositif, certains effets pouvant découler de la formation dans sa globalité (puisqu'elle comprend d'autres activités visant le développement de la réflexivité).

De futures recherches devront donc s'attacher à employer une méthodologie permettant le recueil d'autres types d'informations sur ce dispositif d'analyse des pratiques conçu pour être « réflexif ». La nature du lien entre les composantes réflexives identifiées, la distinction entre processus et effets de la réflexivité, la possibilité de détacher les effets précis du dispositif devront faire lobjet de nouvelles investigations.

\section{Notes}

1 Cadre européen des certifications.

2 Voir http://www.gallilex.cfwb.be/document/pdf/25595_001.pdf.

3 Par exemple, un verbatim de type "l'analyse de certaines situations dépend des personnes qui font cette analyse " a été classé par les chercheuses dans la rubrique "relativité des points de vue».

4 Un verbatim correspond à une unité de sens repérée au sein d'une réponse à une question ouverte dans le questionnaire (exemples : Il existe plusieurs points de vue sur une même situation; on a tous une vision différente du métier selon nos représentations).

5 Pour obtenir davantage d'explications sur « les compétences évoquées », le lecteur est renvoyé à la section 3.

6 Ces catégories sont citées en partant de la catégorie évoquée par un plus grand nombre d’étudiants (compétence de description, chiffre romain I) à la catégorie évoquée par un moins grand nombre d'étudiants au sein des questionnaires (prise de conscience de la contextualisation de la situation, chiffre romain V). Les chiffres romains de I à V décrivent lordre de ces catégories dans le tableau.

7 Bien que ce point ne sera pas développé ici, notons deux hypothèses qui pourraient être émises quant à la faible présence de la compétence de problématisation au sein des questionnaires. En premier lieu, il est probable que les étudiants disposent de moins de mots pour se référer à cette étape parce qu'ils n'en perçoivent peut-être pas la spécificité. En second lieu, cette étape occupant moins de temps dans la séance que les autres étapes, l’attention et la mémoire en sont moins frappées.

\section{Références}

Bertone, S., Chaliès, S., et Clot, Y. (2009). Contribution d'une théorie de l'action à la conceptualisation et à l'évaluation des pratiques réflexives dans les dispositifs de formation initiale des enseignants. Le travail humain, 72(2), 105-125. http:// dx.doi.org/10.3917/th.722.0105

Buysse, A. et Vanhulle, S. (2009). Écriture réflexive et développement professionnel : quels indicateurs? Questions vives, 5(11), 225-242. http://dx.doi.org/10.4000/questionsvives.603 
Charlier, E., Beckers, J., Boucenna, S., Biémar, S., François, N. et Leroy, C. (2013). Comment soutenir la démarche réflexive? Bruxelles : De Boeck.

Donnay, J. et Charlier, E. (2006). Apprendre par l'analyse des pratiques : Initiation au compagnonnage réflexif. Namur : Presses universitaires de Namur.

Gaudin, C. et Chaliès, S. (2012). L'utilisation de la vidéo dans la formation professionnelle des enseignants novices. Revue française de pédagogie, (178), 115-130. http://dx.doi.org/10.4000/rfp.3590

Ghaye, T. (2006). Developing the reflective healthcare team. Oxford : Wiley-Blackwell.

Maroy, C. (2001). Le modèle du praticien réflexif à l'épreuve de l'enquête. Cahier de recherche du GIRSEF, (12), 1-26. Repéré à https://halshs.archives-ouvertes.fr/halshs-00603562

Perrenoud, P. (2004). Adosser la pratique réflexive aux sciences sociales, condition de la professionnalisation. Éducation permanente, (160), 35-60.

Perrenoud, P. (2005). Assumer une identité réflexive. Éducateur, 2, 30-33.

Ria, L. (2018). Conception de laboratoires d'analyse vidéo de l'activité enseignante en établissement scolaire. Dans C. Van Nieuwenhoven, S. Colognesi et S. Beausaert (dir.), Accompagner les pratiques des enseignants, un défipour le développement professionnel en formation initiale, en insertion et en cours de carrière (p. 205-218). Louvain-la-Neuve : Presses universitaires de Louvain.

Ria, L. et Leblanc, S. (2011). Conception de la plateforme de formation Néopass@ction à partir d'un observatoire de l'activité des enseignants débutants : enjeux et processus. Activités, 8(2). http://dx.doi.org/10.4000/activites.2618

Rutman, L. (1982). Planification d'une étude évaluative. Dans R. Lecomte et L. Rutman (dir.), Introduction aux méthodes de recherche évaluative (p. 23-46). Québec, QC : Presses de l'Université Laval.

Scaife, J. (2010). Supervising the reflective practitioner. Londres : Routledge.

Schön, D. A. (1983). The reflective practioner: how professionals think in action. New York, NY : Basic Books.

Schön, D. A. (1994). Le praticien réflexif: à la recherche du savoir caché dans l'agir professionnel. Montréal, QC : Logiques.

Tardif, M., Borges, C. et Malo, A. (2012). Le virage réflexif en éducation. Bruxelles : De Boeck.

Trinquier, M.-P. (2014). Pratiques professionnelles. Dans A. Jorro (dir.), Dictionnaire des concepts de la professionnalisation (p. 221-228). Bruxelles : De Boeck.

Vacher, Y. (2015). Construire une pratique réflexive : comprendre et agir. Bruxelles : De Boeck. http://dx.doi.org/10.3917/dbu.vachr.2015.01

Van der Maren, J.-M. (1993). Savoirs enseignants et professionnalisation de l'enseignement. Revue des sciences de l'éducation, 19(1), 159-173. http://dx.doi.org/10.7202/031605ar

Wittorski, R. (2008). La professionnalisation. Savoirs, (17), 9-36. http://dx.doi.org/10.3917/savo.017.0009

Yvon, F. et Clot, Y. (2003). Apprentissage et développement dans le travail enseignant. Pratiques psychologiques, 1, 17-33.

\section{Pour citer cet article}

Fischer, L., Dantinne, F. et Charlier, E. (2019). Évaluation des effets d'un dispositif de formation initiale visant à susciter la réflexivité chez les futurs enseignants. Fomation et profession. 27(2), 45-57. http://dx.doi.org/10.18162/fp.2019.494 